\title{
APPENDIX
}

\section{The Fully-Liquid Case.}

Setting the expressions for $P_{t}$ in Equations (5) and (7) equal to each other, and similarly for the expressions for $Q_{t}$ in Equations (6) and (8), implies the following pair of equations:

$$
\begin{aligned}
& 0=E_{t}\left[\int_{0}^{\infty}\left\{e^{-\beta s}\left(\frac{C_{t}}{C_{t+s}}\right)-e^{-\delta s}\left(\frac{X_{t}+Y_{t}-C_{t}}{X_{t+s}+Y_{t+s}-C_{t+s}}\right)\right\} X_{t+s} d s\right], \\
& 0=E_{t}\left[\int_{0}^{\infty}\left\{e^{-\beta s}\left(\frac{C_{t}}{C_{t+s}}\right)-e^{-\delta s}\left(\frac{X_{t}+Y_{t}-C_{t}}{X_{t+s}+Y_{t+s}-C_{t+s}}\right)\right\} Y_{t+s} d s\right] .
\end{aligned}
$$

Both of the above expressions are satisfied by requiring that

$$
e^{-\beta s}\left(\frac{C_{t}}{C_{t+s}}\right)=e^{-\delta s}\left(\frac{X_{t}+Y_{t}-C_{t}}{X_{t+s}+Y_{t+s}-C_{t+s}}\right),
$$

hold for all $s, X_{t+s}$, and $Y_{t+s}$. Solving this expression for $C_{t+s}$ implies

$$
C_{t+s}=\frac{e^{-\beta s} C_{t}}{e^{-\beta s} C_{t}+e^{-\delta s}\left(X_{t}+Y_{t}-C_{t}\right)}\left(X_{t+s}+Y_{t+s}\right) .
$$

Thus, $C_{t+s}$ can be expressed in terms of $C_{t}$.

Similarly, satisfying the first-order conditions as of time zero requires that

$$
\begin{aligned}
C_{t} & =\frac{e^{-\beta t} C}{e^{-\beta t} C+e^{-\delta t}(X+Y-C)}\left(X_{t}+Y_{t}\right), \\
C_{t+s} & =\frac{e^{-\beta(t+s)} C}{e^{-\beta(t+s)} C+e^{-\delta(t+s)}(X+Y-C)}\left(X_{t+s}+Y_{t+s}\right) .
\end{aligned}
$$

Substituting the expression for $C_{t}$ in Equation (A5) into Equation (A4), however, reduces Equation (A4) to Equation (A6). Thus, requiring that Equation (A5) hold for all $t$ is sufficient for the first-order conditions in Equations (5) through (8) to be satisfied for all $t$.

Dividing $C_{t}$ by the expression for $C_{t+s}$ in Equation (A4) and rearranging gives

$$
\left(\frac{C_{t}}{C_{t+s}}\right)=\frac{C_{t}+\left(X_{t}+Y_{t}-C_{t}\right) e^{(\beta-\delta) s}}{X_{t+s}+Y_{t+s}} .
$$

Substituting this into Equations (5) and (6) and rearranging yields

$$
\begin{aligned}
P_{t}=C_{t} E_{t}\left[\int_{0}^{\infty} e^{-\beta s}\right. & \left.\left(\frac{X_{t+s}}{X_{t+s}+Y_{t+s}}\right) d s\right] \\
& +\left(X_{t}+Y_{t}-C_{t}\right) E_{t}\left[\int_{0}^{\infty} e^{-\delta s}\left(\frac{X_{t+s}}{X_{t+s}+Y_{t+s}}\right) d s\right], \\
Q_{t}=C_{t} E\left[\int_{0}^{\infty} e^{-\beta s}\right. & \left.\left(\frac{Y_{t+s}}{X_{t+s}+Y_{t+s}}\right) d s\right] \\
& +\left(X_{t}+Y_{t}-C_{t}\right) E_{t}\left[\int_{0}^{\infty} e^{-\delta s}\left(\frac{Y_{t+s}}{X_{t+s}+Y_{t+s}}\right) d s\right] .
\end{aligned}
$$


Section 3 of this Appendix shows that these equations can be reexpressed as

$$
\begin{gathered}
P_{t}=C_{t} A\left(\beta, X_{t}, Y_{t}\right)+\left(X_{t}+Y_{t}-C_{t}\right) A\left(\delta, X_{t}, Y_{t}\right), \\
Q_{t}=C_{t} B\left(\beta, X_{t}, Y_{t}\right)+\left(X_{t}+Y_{t}-C_{t}\right) \quad B\left(\delta, X_{t}, Y_{t}\right),
\end{gathered}
$$

which are Equations (11) and (12).

To solve for $C$, note that after consuming at time zero, the first agent's wealth equals $w(P+X+Q+$ $Y)-C$, where the first term represents the value of the agent's endowment (with dividends). Setting the value of the agent's wealth equal to the present value of his future consumption stream gives

$$
\begin{aligned}
w(P+X+Q+Y)-C & =E\left[\int_{0}^{\infty} e^{-\beta t}\left(\frac{C}{C_{t}}\right) C_{t} d t\right], \\
& =C / \beta,
\end{aligned}
$$

which implies

$$
C=\frac{w(P+X+Q+Y)}{1+\frac{1}{\beta}}
$$

Substituting in the expressions for $P$ and $Q$ from Equations (A8) and (A9), and solving for $C$ gives

$$
C=\frac{w \beta(1+\delta)}{\delta(1+\beta)+w(\beta-\delta)}(X+Y)
$$

Substituting this expression into Equation (A5) gives Equation (9).

From Equation (9), optimal consumption is homogeneous of degree one in total dividends $X_{t}+Y_{t}$. Based on this, we conjecture (and later verify) that the dynamic portfolio strategy that generates $C_{t}$ consists of equal numbers of shares of the two assets, $N_{t}=M_{t}$, where $N_{t}$ is a differentiable function of time. By definition, consumption equals the sum of dividends received minus net purchases of assets. Thus,

$$
C_{t}=N_{t}\left(X_{t}+Y_{t}\right)-\left(P_{t}+Q_{t}\right) N_{t}^{\prime}
$$

where $N_{t}^{\prime}$ denotes a derivative. From Equations (A8) and (A9) it follows that

$$
P_{t}+Q_{t}=\frac{C_{t}}{\beta}+\frac{X_{t}+Y_{t}-C_{t}}{\delta} .
$$

Substituting this and the expression for $C_{t}$ in Equation (A5) into Equation (A16) gives the ordinary differential equation

$$
N_{t}^{\prime}-\left(\frac{\beta \delta\left(C e^{\delta t}+(X+Y-C) e^{\beta t}\right)}{C \delta e^{\delta t}+\beta(X+Y-C) e^{\beta t}}\right) N_{t}=\frac{-C \beta \delta e^{\delta t}}{C \delta e^{\delta t}+\beta(X+Y-C) e^{\beta t}} .
$$

This is a standard first-order linear differential equation which can be solved directly by an integration. The initial value of $N$ is determined by imposing the condition that $N(P+Q)$ equals the first agent's initial wealth after time-zero consumption. From Spiegel (1967), the solution to this differential equation is the 
expression given in Equation (10). This verifies the conjecture and also establishes that the consumption strategy identified in Equation (A5) is feasible.

In solving the illiquid-asset problem, it is also necessary to solve for $C_{T}$ when the first agent has $w$ shares of the first asset and $v$ shares of the second asset at time $T$ (instead of $w$ shares of each). Since the model reverts back to the fully-liquid case at time $T$ (with the exception that there is no consumption gulp at time $T$ ), the expressions for $P_{T}$ and $Q_{T}$ are as given from Equations (11) and (12). To solve for $C_{T}$ in this more general case, we set the value of the first agent's wealth at time $T$ equal to the present value of his remaining consumption stream

$$
\begin{aligned}
w P_{T}+v Q_{T} & =E_{T}\left[\int_{0}^{\infty} e^{-\beta s}\left(\frac{C_{T}}{C_{T+s}}\right) C_{T+s} d s\right], \\
& =C_{T} / \beta
\end{aligned}
$$

which implies

$$
C_{T}=\beta\left(w P_{T}+v Q_{T}\right)
$$

Substituting in the expressions for $P_{T}$ and $Q_{T}$ in Equations (A10) and (A11), and then solving for $C_{T}$ gives,

$$
C_{T}=\frac{\left(w A\left(\delta, X_{T}, Y_{T}\right)+v B\left(\delta, X_{T}, Y_{T}\right)\right)\left(X_{T}+Y_{T}\right)}{\frac{1}{\beta}+w\left(A\left(\delta, X_{T}, Y_{T}\right)-A\left(\beta, X_{T}, Y_{T}\right)\right)+v\left(B\left(\delta, X_{T}, Y_{T}\right)-B\left(\beta, X_{T}, Y_{T}\right)\right)}
$$

in this general case. In the illiquid-asset case, $C_{T}$ is given by substituting in $w=N_{T}$ and $v=M$ into the above equation. In the illiquid-market case, $C_{T}$ is given by substituting in $w=N$ and $v=M$ into the above equation.

\section{The Illiquid-Asset Case.}

To solve the illiquid-asset case numerically, we first discretize the problem by approximating the dividend dynamics using binomial processes. Let $\Delta t$ denote the discretization step. Then,

$$
\begin{aligned}
X_{t+\Delta t} & =X_{t} \exp \left(\left(\mu_{X}-\sigma_{X}^{2} / 2\right) \Delta t \pm \sigma_{X} \sqrt{\Delta t}\right), \\
Y_{t+\Delta t} & =Y_{t} \exp \left(\left(\mu_{Y}-\sigma_{Y}^{2} / 2\right) \Delta t \pm \sigma_{Y} \sqrt{\Delta t}\right),
\end{aligned}
$$

where the probability of both processes increasing or decreasing together is $p / 2$, the probability of the first process increasing and the second decreasing is $(1-p) / 2$, the probability of the first process decreasing and the second increasing is $(1-p) / 2$, and where $p=(1+\rho) / 2$. This assumption insures that the correlation between the two processes is $\rho$. Note that we use this binomial process merely as an approximation to the continuous-time process.

To simplify the exposition, we make the assumption that $\Delta t=1$. The numerical procedure, however, is applicable to any positive value of $\Delta t$. Note that at time $t$, where $0<t<T$, the first agent' consumption is

$$
C_{t}=N_{t-1} X_{t}+M Y_{t}-P_{t}\left(N_{t}-N_{t-1}\right) .
$$

The recursive approach begins by first solving the problem at time $T-1$, conditional on knowing the functional forms of $P_{T}$ and $C_{T}$. Since the model reverts back to the fully-liquid model at time $T$, however, these functional forms are those for the fully-liquid model. At time $T-1$, the first-order conditions of optimality for the two agents are 


$$
\begin{array}{r}
\frac{P_{T-1}}{C_{T-1}}=E_{T-1}\left[\frac{e^{-\beta}\left(X_{T}+P_{T}\right)}{C_{T}}\right], \\
\frac{P_{T-1}}{X_{T-1}+Y_{T-1}-C_{T-1}}=E_{T-1}\left[\frac{e^{-\delta}\left(X_{T}+P_{T}\right)}{X_{T}+Y_{T}-C_{T}}\right] .
\end{array}
$$

Substituting in the expression for $C_{T-1}$ from Equation (A25) into Equations (A26) and (A27) and solving for $P_{T-1}$ gives the following pair of equations,

$$
\begin{aligned}
P_{T-1}= & \frac{\left(N_{T-2} X_{T-1}+M Y_{T-1}\right) E_{T-1}\left[\frac{e^{-\beta}\left(X_{T}+P_{T}\right)}{C_{T}}\right]}{1+\left(N_{T-1}-N_{T-2}\right) E_{T-1}\left[\frac{e^{-\beta}\left(X_{T}+P_{T}\right)}{C_{T}}\right]}, \\
P_{T-1}= & \frac{\left(\left(1-N_{T-2}\right) X_{T-1}+(1-M) Y_{T-1}\right) E_{T-1}\left[\frac{e^{-\delta}\left(X_{T}+P_{T}\right)}{X_{T}+Y_{T}-C_{T}}\right]}{1-\left(N_{T-1}-N_{T-2}\right) E_{T-1}\left[\frac{e^{-\delta}\left(X_{T}+P_{T}\right)}{X_{T}+Y_{T}-C_{T}}\right]} .
\end{aligned}
$$

For a given choice of $M$, and recognizing that both $P_{T}$ and $C_{T}$ can be expressed as explicit functions of $N_{T-1}$, $M, X_{T}$, and $Y_{T}$ (using the generalized solution for consumption in the fully-liquid case given in Equation (A22)), these two equations can be set equal to each other to provide a single equation in the unknown $N_{T-1}$. This single equation is easily solved numerically for $N_{T-1}$, which, in turn, can be substituted back into Equation (A28) or Equation (A29) to give $P_{T-1}$.

To provide a full solution at time $T-1$, we solve this problem repeated for every combination of $X_{T-1}$ and $Y_{T-1}$ on the two-dimensional binomial tree and for every value of $M$ and $N_{T-2}$ ranging from zero to one, (in steps of 0.001). Although computationally intensive, this procedure gives us a "lookup" table from which we can interpolate the equilibrium values of $N_{T-1}$ and $P_{T-1}$ for any value of $X_{T-1}, Y_{T-1}, M$ and $N_{T-2}$.

The algorithm now rolls back to time $T-2$. Exactly as before, the agents first-order conditions as of time $T-2$ can be rearranged to give the pair of equations,

$$
\begin{aligned}
P_{T-2}= & \frac{\left(N_{T-3} X_{T-2}+M Y_{T-2}\right) E_{T-2}\left[\frac{e^{-\beta}\left(X_{T-1}+P_{T-1}\right)}{C_{T-1}}\right]}{1+\left(N_{T-2}-N_{T-3}\right) E_{T-2}\left[\frac{e^{-\beta}\left(X_{T-1}+P_{T-1}\right)}{C_{T-1}}\right]}, \\
P_{T-2}= & \frac{\left(\left(1-N_{T-3}\right) X_{T-2}+(1-M) Y_{T-2}\right) E_{T-2}\left[\frac{e^{-\delta}\left(X_{T-1}+P_{T-1}\right)}{X_{T-1}+Y_{T-1}-C_{T-1}}\right]}{1-\left(N_{T-2}-N_{T-3}\right) E_{T-2}\left[\frac{e^{-\delta}\left(X_{T-1}+P_{T-1}\right)}{X_{T-1}+Y_{T-1}-C_{T-1}}\right]} .
\end{aligned}
$$

These can again be set equal and solved for $N_{T-2}$ (and $P_{T-2}$ ) for each set of values for $X_{T-2}, Y_{T-2}, M$, and $N_{T-3}$, given the functional forms for $P_{T-1}$ and $C_{T-1}$. These functional forms, however, can be approximated directly using the lookup table constructed in the previous step (the value of $C_{T-1}$ follows from the value of $N_{T-1}$ because of Equation (A25)). Once again, we solve the problem at time $T-2$ repeatedly and construct a new lookup table for $N_{T-2}$ and $P_{T-2}$. This process iterates back until we have constructed a lookup table for time one.

At time zero, the first-order conditions for the agents are

$$
\begin{array}{r}
\frac{P}{C}=E\left[\frac{e^{-\beta}\left(X_{1}+P_{1}\right)}{C_{1}}\right], \\
\frac{P}{X+Y-C}=E\left[\frac{e^{-\delta}\left(X_{1}+P_{1}\right)}{X_{1}+Y_{1}-C_{1}}\right],
\end{array}
$$




$$
\begin{gathered}
\frac{Q}{C}=E\left[\sum_{i=1}^{T} e^{-\beta i} \frac{Y_{i}}{C_{i}}+e^{-\beta T} \frac{Q_{T}}{C_{T}}\right], \\
\frac{Q}{X+Y-C}=E\left[\sum_{i=1}^{T} e^{-\delta i} \frac{Y_{i}}{X_{i}+Y_{i}-C_{i}}+e^{-\delta T} \frac{Q_{T}}{X_{T}+Y_{T}-C_{T}}\right] .
\end{gathered}
$$

Since both assets can be traded at time zero,

$$
C=w(X+Y+P+Q)-P N-Q M
$$

By substituting this expression into Equations (A32) through (A35), the values $P$ and $Q$ can be eliminated, resulting in a system of two equations in the two unknowns $M$ and $N$. Again, this system is straightforward to solve numerically. Once $N$ and $M$ are determined, the values of $P$ and $Q$ are given directly by substituting $N$ and $M$ back into the first-order conditions.

To minimize the computational burden, we make a number of simplifying assumptions in implementing the algorithm. First, we set $\Delta t$ equal to one year (except when we solve the model for an illiquidity horizon of one year, in which case $\Delta t$ equals six months). Second, we model the binomial process and value the cash flows out only to 100 years. Third, rather than using the closed-form solutions for the fully-liquid case, we solve for these numerically using the same binomial grid. This allows us to compare the fully-liquid case directly with the illiquid-asset case without introducing small discretization errors. Finally, rather than evaluating the expectations on the right hand side of Equations (A34) and (A35) directly as of time zero, we use a similar type of lookup table approach to solve for the values of the expectations sequentially from time $T-1$ to time zero (using the double expectation theorem repeatedly).

Finally, since an unconstrained equilibrium may not exist, it is important to consider how the algorithm should be modified in this situation. First, it is important to recognize that agents will never take a short position in the liquid asset at time $0<t<T$. The reason for this is that the dividends for the illiquid asset could decline to the point where an agent's consumption became negative, and therefore, his utility became negative infinity. Thus, zero and one are upper and lower bounds for $N_{t}$. Now consider the situation where the first agent has a lower valuation for the liquid asset even when $N_{t}=0$. Since the first agent cannot sell any more shares to the second agent, his first-order conditions cannot be satisfied. In this situation, $N_{t}=0$, and $P_{t}$ is given from the second agent's first-order conditions. A similar argument holds for the second agent.

Now consider the situation at time zero. Similar reasoning to the above shows that agents will not take short positions in either asset at time zero. Thus, zero and one become upper and lower bounds for $N$ and $M$. If one agent has a lower valuation for an asset even when his holdings of the asset are zero, then that agent's first-order condition for the asset cannot be satisfied. In this situation, we use the first-order condition for the other agent in determining the equilibrium values for the other portfolio weight and for the asset prices at time zero.

3. The $A\left(\cdot, X_{t}, Y_{t}\right)$ and $B\left(\cdot, X_{t}, Y_{t}\right)$ Functions.

We define $A\left(c, X_{t}, Y_{t}\right)$ as the expectation

$$
A\left(c, X_{t}, Y_{t}\right)=E_{t}\left[\int_{0}^{\infty} e^{-c s}\left(\frac{X_{t+s}}{X_{t+s}+Y_{t+s}}\right) d s\right]
$$

that appears in various forms in the agents' first-order conditions. This can be rewritten as

$$
E_{t}\left[\int_{0}^{\infty} e^{-c s}\left(\frac{1}{1+q e^{u}}\right) d s\right]
$$

where $q=\frac{Y_{t}}{X_{t}}$, and $u$ is a normally distributed random variable with mean $\mu s$ and variance $\sigma^{2} s$, where 


$$
\begin{aligned}
\mu & =\mu_{Y}-\mu_{X}-\sigma_{Y}^{2} / 2+\sigma_{X}^{2} / 2, \\
\sigma^{2} & =\sigma_{X}^{2}+\sigma_{Y}^{2}-2 \rho \sigma_{X} \sigma_{Y} .
\end{aligned}
$$

Introducing the density for $u$ into the above expectation gives

$$
\int_{0}^{\infty} \int_{-\infty}^{\infty} e^{-c s} \frac{1}{\sqrt{2 \pi \sigma^{2} s}} \frac{1}{1+q e^{u}} \exp \left(\frac{-(u-\mu s)^{2}}{2 \sigma^{2} s}\right) d u d s
$$

Interchanging the order of integration and collecting terms in $s$ gives,

$$
\int_{-\infty}^{\infty} \frac{1}{\sqrt{2 \pi \sigma^{2}}} \frac{1}{1+q e^{u}} \exp \left(\frac{\mu u}{\sigma^{2}}\right) \int_{0}^{\infty} s^{-1 / 2} \exp \left(-\frac{u^{2}}{2 \sigma^{2}} \frac{1}{s}-\frac{\mu^{2}+2 c \sigma^{2}}{2 \sigma^{2}} s\right) d s d u
$$

From Equation (3.471.9) of Gradshteyn and Ryzhik (2000), this expression becomes,

$$
\int_{-\infty}^{\infty} \frac{2}{\sqrt{2 \pi \sigma^{2}}} \frac{1}{1+q e^{u}} \exp \left(\frac{\mu u}{\sigma^{2}}\right)\left(\frac{u^{2}}{\mu^{2}+2 c \sigma^{2}}\right)^{1 / 4} K_{1 / 2}\left(2 \sqrt{\frac{u^{2}\left(\mu^{2}+2 c \sigma^{2}\right)}{4 \sigma^{4}}}\right) d u
$$

where $K_{1 / 2}(\cdot)$ is the modified Bessel function of order 1/2 (see Abramowitz and Stegum (1970) Chapter 9). From the identity relations for Bessel functions of order equal to an integer plus one half given in Gradshteyn and Ryzhik Equation (8.469.3), however, the above expression can be expressed as,

$$
\frac{1}{\psi} \int_{-\infty}^{\infty} \frac{1}{1+q e^{u}} \exp \left(\frac{\mu u}{\sigma^{2}}\right) \exp \left(-\frac{\psi}{\sigma^{2}}|u|\right) d u
$$

where

$$
\psi=\sqrt{\mu^{2}+2 c \sigma^{2}}
$$

In turn, Equation (A44) can be written

$$
\frac{1}{\psi} \int_{0}^{\infty} \frac{1}{1+q e^{u}} \exp (\gamma u) d u+\frac{1}{\psi} \int_{-\infty}^{0} \frac{1}{1+q e^{u}} \exp (\theta u) d u
$$

where

$$
\gamma=\frac{\mu-\psi}{\sigma^{2}}, \quad \theta=\frac{\mu+\psi}{\sigma^{2}}
$$

Define $y=e^{-u}$. By a change of variables Equation (A37) can be written

$$
\frac{1}{q \psi} \int_{0}^{1} \frac{1}{1+y / q} y^{-\gamma} d y+\frac{1}{\psi} \int_{0}^{1} \frac{1}{1+q y} y^{\theta-1} d y .
$$

From Abramowitz and Stegum Equation (15.3.1), this expression becomes

$$
\frac{1}{q \psi(1-\gamma)} F(1,1-\gamma ; 2-\gamma ;-1 / q)+\frac{1}{\psi \theta} F(1, \theta ; 1+\theta ;-q)
$$


Substituting in for $q$ gives the expression for $A\left(c, X_{t}, Y_{t}\right)$

$$
A\left(c, X_{t}, Y_{t}\right)=k_{1} \quad\left(X_{t} / Y_{t}\right) \quad F\left(1,1-\gamma ; 2-\gamma ;-\frac{X_{t}}{Y_{t}}\right)+k_{2} \quad F\left(1, \theta ; 1+\theta ;-\frac{Y_{t}}{X_{t}}\right),
$$

where

$$
k_{1}=\frac{1}{\psi(1-\gamma)}, \quad k_{2}=\frac{1}{\psi \theta} .
$$

The function $B\left(c, X_{t}, Y_{t}\right)$ is defined as the expectation,

$$
B\left(c, X_{t}, Y_{t}\right)=E_{t}\left[\int_{0}^{\infty} e^{-c s}\left(\frac{Y_{t+s}}{X_{t+s}+Y_{t+s}}\right) d s\right]
$$

The evaluation of this expectation is omitted since it is virtually the same at that for $A\left(c, X_{t}, Y_{t}\right)$ above. The resulting expression for $B\left(c, X_{t}, Y_{t}\right)$ is

$$
B\left(c, X_{t}, Y_{t}\right)=k_{3} \quad\left(Y_{t} / X_{t}\right) \quad F\left(1,1+\theta ; 2+\theta ;-\frac{Y_{t}}{X_{t}}\right)-k_{4} \quad F\left(1,-\gamma ; 1-\gamma ;-\frac{X_{t}}{Y_{t}}\right)
$$

where

$$
k_{3}=\frac{1}{\psi(1+\theta)}, \quad k_{4}=\frac{1}{\psi \gamma} .
$$

Substituting the solutions for $A\left(c, X_{t}, Y_{t}\right)$ and $B\left(c, X_{t}, Y_{t}\right)$ into Equations (A8) and (A9) gives the expressions for $P_{t}$ and $Q_{t}$ in Equations (11) and (12). 